\title{
Anti-inflammatory activity of Kochia scoparia fruit on contact dermatitis in mice
}

\author{
SUZY JO $^{1 *}$, JUNGHYUN RYU $^{1 *}$, HYE-YEON HAN $^{2}$, GEUMSAN LEE $^{3}$, MI HEON RYU ${ }^{2}$ and HYUNGWOO KIM ${ }^{1}$ \\ ${ }^{1}$ Department of Pharmacology, School of Korean Medicine; ${ }^{2}$ Department of Oral Pathology, School of Dentistry, \\ Pusan National University, Yangsan, Gyeongsangnam-do 626-870; ${ }^{3}$ Department of Herbology, College of Korean Medicine, \\ Wonkwang University, Iksan, Jeollabuk-do 565-806, Republic of Korea
}

Received March 2, 2015; Accepted November 17, 2015

DOI: $10.3892 / \mathrm{mmr} .2015 .4698$

\begin{abstract}
The mature fruit of Kochia scoparia (L.) Schrad. is widely administered in China and Korea as a medicinal herb for treatment of skin diseases, diabetes mellitus and rheumatoid arthritis. The present study investigated the effects of methanol extracts of $K$. scoparia dried fruit (MEKS) on ear swelling, histopathological changes (such as epidermal acanthosis, spongiosis and immune cell infiltration) and cytokine production in 1-fluoro-2,4-dinitrofluorobenzene (DNFB)-induced contact dermatitis mice. Topical application of MEKS inhibited DNFB-induced ear thickness and weight increases, as well as DNFB-induced epidermal acanthosis, spongiosis and immune cell infiltration. In addition, treatment with MEKS significantly decreased the levels of tumor necrosis factor- $\alpha$, interferon- $\gamma$ and monocyte chemotactic protein-1 in inflamed tissues. These data indicate that the mature fruit of $K$. scoparia has the potential to be administered for the treatment of inflammatory skin diseases and that the anti-inflammatory action of $K$. scoparia is involved in the inhibition of type $1 \mathrm{~T}$ helper cell skewing reactions.
\end{abstract}

\section{Introduction}

Contact dermatitis (CD), the predominant inflammatory skin disease worldwide, is a type of eczematous eruption that occurs following contact with foreign substances $(1,2)$. The histopathological features of CD include epithelial hyperkeratosis, acanthosis and spongiosis that present with perivascular inflammatory cell infiltration, primarily consisting of $\mathrm{T}$ cells in the epidermis and upper dermis (3).

Correspondence to: Professor Hyungwoo Kim, Department of Pharmacology, School of Korean Medicine, Pusan National University, 49 Busandaehak-ro, Yangsan, Gyeongsangnam-do 626-870, Republic of Korea

E-mail:kronos7@pusan.ac.kr

*Contributed equally

Key words: Kochia scoparia, contact dermatitis, cytokine, inflammation
Following exposure to a skin irritant, various cells, including activated keratinocytes, dendritic cells, dermal fibroblasts, and endothelial cells, release pro-inflammatory and inflammatory cytokines/chemokines (4). The cytokines/chemokines induce the dilatation of blood vessels and inflammatory cell infiltration, resulting in epidermal acanthosis and spongiosis, however, the precise mechanism remains to be elucidated (4). Tumor necrosis factor (TNF)- $\alpha$, interferon (IFN) $-\gamma$ and monocyte chemotactic protein (MCP)-1 are key primary cytokines in the activation cascade and pathogenesis of CD $(4,5)$.

The fruit of Kochia scoparia (L.) Schrad., which is also designated Bassia scoparia, Bassia sieversiana or Kochia alata, is administered to treat skin diseases, diabetes mellitus and rheumatoid arthritis in Chinese and Korean traditional medicine $(6,7)$. Furthermore, it is frequently administered to treat urticaria in Taiwan (8). Previous studies have indicated that $K$. scoparia and its components exert anti-inflammatory (9) and anti-allergic $(10,11)$ activities. Choi et al (12) recently reported that the water extract of $K$. scoparia inhibits the development of $\mathrm{CD}$ in mice.

The aim of the current study was to evaluate the anti-inflammatory effects of methanol extracts of $K$. scoparia dried fruit (MEKS) on 1-fluoro-2,4-dinitrobenzene (DNFB)-induced CD. For this reason, the effects of MEKS on ear thickness and weight, the histopathological changes in ear tissue samples, and the cytokine and chemokine levels of inflamed tissues were assessed in vivo.

\section{Materials and methods}

Preparation of MEKS. The mature fruit of $K$. scoparia was purchased from Hwalim Medicinal Herbs (Pusan, Korea). A total of $100 \mathrm{~g} \mathrm{~K}$. scoparia dried fruit was immersed in $1,000 \mathrm{ml}$ methanol and sonicated for $30 \mathrm{~min}$, following which the samples were extracted for $48 \mathrm{~h}$. The extract was subsequently filtered through Whatman filter paper No. 20 (Advantech, Milpitas, CA, USA) and evaporated under reduced pressure using a vacuum evaporator (N-1000V-W; Eyela Co., Ltd., Tokyo, Japan), following which the condensed extract was lyophilized using a freeze dryer (Labconco Corporation, Kansas City, MO, USA) and 4.46 g lyophilized powder was obtained (yield, 4.46\%). An aliquot of the extract (MEKS) 
was deposited at the Department of Pharmacology, School of Korean Medicine, Pusan National University (Yangsan, South Korea; voucher no. MH2013-006).

Animals. A total of 44 male 6-week-old BALB/c mice were purchased from Samtaco (Incheon, Korea). The mice were housed under specific pathogen-free conditions with a 12-h light/dark cycle and free access to standard rodent food and water. All animal experiments were approved by the Animal Care and Use Committee of Pusan National University and performed according to institutional guidelines (PNU-2011-000406).

Induction of CD and experimental design. Mice were sensitized by applying $50 \mu 1$ DNFB (0.1\%, v/v; Sigma-Aldrich, St. Louis, MO, USA), in a vehicle of acetone:olive oil (AOO; 4:1), onto the shaved back of each mouse for three consecutive days. Four days following sensitization, each mouse was challenged by applying $30 \mu \mathrm{l}$ DNFB $(0.2 \%, \mathrm{v} / \mathrm{v})$ in AOO onto the dorsal surface of each ear every two days (four applications in total). MEKS solution (30, 100 or $300 \mu \mathrm{g} /$ ear) was applied onto the dorsal surface of each ear for seven consecutive days. Healthy mice $(n=6)$ were treated with the vehicle, AOO and only AOO was topically applied (non-treated normal mice; NOR). Control mice $(n=8)$ were sensitized and challenged with DNFB, following which AOO was topically applied (non-treated CD mice; CTL). MEKS-treated mice $(n=8)$ were sensitized and challenged with DNFB, and 30, 100 or $300 \mu \mathrm{g} / \mathrm{ear}(1 \%$, w/v) of MEKS was topically applied. Dexamethasone (DEX; Sigma-Aldrich)-treated mice $(\mathrm{n}=6)$ were sensitized and challenged with DNFB, exposed to $75 \mu \mathrm{g} /$ ear DEX and served as a positive control. The experimental design is summarized in Fig. 1.

Measurement of ear thicknesses and weights. Mice were anesthetized with $30 \mathrm{mg} / \mathrm{kg}$ Zoletil ${ }^{\circledR}$ (Virbac, Carros, France) and sacrificed by cervical dislocation, following which the thickness of each ear was measured using vernier calipers (Mitutoyo, Kanagawa, Japan). The pieces of ear (5 $\mathrm{mm}$ in diameter) obtained via dermal punch were weighed using a microbalance (US/EL-2000S; Sartorius AG, Göttingen, Germany).

Histopathological examination. Following assessment of ear thicknesses and weights, ear tissues $(4 \mu \mathrm{m})$ were resected, formalin-fixed (Sigma-Aldrich) and embedded in paraffin (Leica Microsystems GmbH, Wetzlar, Germany). Sections were cut and stained with hematoxylin and eosin (Sigma-Aldrich) to observe histopathological changes, such as epidermal acanthosis, spongiosis and immune cell infiltration. Stained tissue sections were observed using a light microscope (magnification, x200; DE/Axio Imager A1; Carl Zeiss AG, Oberkochen, Germany).

Evaluation of epidermal acanthosis and immune cell infiltration. To evaluate the epidermal acanthosis and immune cell infiltration, five non-overlapping fields per slide were randomly selected and images were captured with the light microscope. To measure the thickness of the epithelium, the vertical length between the basal lamina and top of

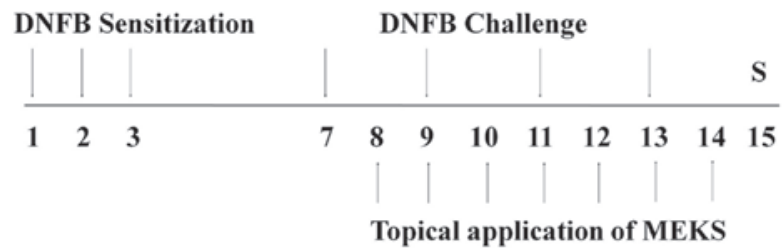

Figure 1. Experimental design. On days 1, 2 and 3 DNFB was applied to the shaved backs of mice in the treatment groups for sensitization. DNFB was then applied to the dorsal surface of each ear on days 7, 9, 11 and 13. MEKS and dexamethasone were topically applied for 7 consecutive days. All animals were sacrificed on day 15. DNFB, 1-fluoro-2,4-dinitrofluorobenzene; MEKS, methanol extracts of Kochia scoparia dried fruit; S, sacrifice.

the outermost stratum granulosum was quantified. For each slide, five lengths were measured at random using Motic Images Plus 2.0 (Motic Instruments, Richmond, BC, Canada), following which the mean epithelial thicknesses of all experimental groups were used for analysis. To evaluate immune cell infiltration, the immune cells were counted using a cell counting grid.

Measurement of cytokine production. Cytokine levels in the ear tissue samples were measured according to the cytometric bead array (CBA) method with the Mouse Inflammation CBA kit (BD Biosciences, San Jose, CA, USA). Resected ear tissue samples were lysed and homogenized with protein extraction solution (Pro-Prep; Intron Biotechnology, Seoul, Korea) using a bullet blender (BB2516; Next Advance, Averill Park, NY, USA) to obtain tissue lysates. The levels of TNF- $\alpha$, IFN- $\gamma$, interleukin (IL)-10 and MCP-1 were measured in $50 \mu \mathrm{g}$ each lysate using the CBA kit. All experimental procedures were conducted according to the manufacturer's protocols.

Measurement of body and spleen weights. Body and spleen weights were measured on day 15 using the microbalance. The effects of MEKS on changes in spleen weights were analyzed as the spleen/body weight ratio.

Statistical analysis. A Mann-Whitney U test was used for all statistical comparisons, and Prism 5 for Windows version 5.01 (GraphPad Software Inc., La Jolla, CA, USA) was used for all analyses. All data are presented as the mean \pm standard deviation and $\mathrm{P}<0.05$ was considered to indicate a statistically significant difference.

\section{Results}

MEKS decreased the DNFB-induced changes in ear thickness and weight. Topical application of DNFB induced ear swelling, which is a major feature of $\mathrm{CD}$. These increases in the thickness and weight of ear tissues were inhibited in a dose-dependent manner by topical application of MEKS (ear thickness: $\mathrm{P}<0.001$, MEKS treatment on left ear; $\mathrm{P}<0.01$, MEKS treatment on right ear; $\mathrm{P}<0.001$, DEX treatment on both ears. Ear weight: $\mathrm{P}<0.05$, 100 or $300 \mu \mathrm{g} / \mathrm{ear}$ MEKS treatment; $\mathrm{P}<0.001,75 \mu \mathrm{g} / \mathrm{ear}$ DEX treatment) as presented in Fig. 2.

MEKS inhibited epidermal spongiosis in the inflamed tissue. Histological examination in the tissue sections of 

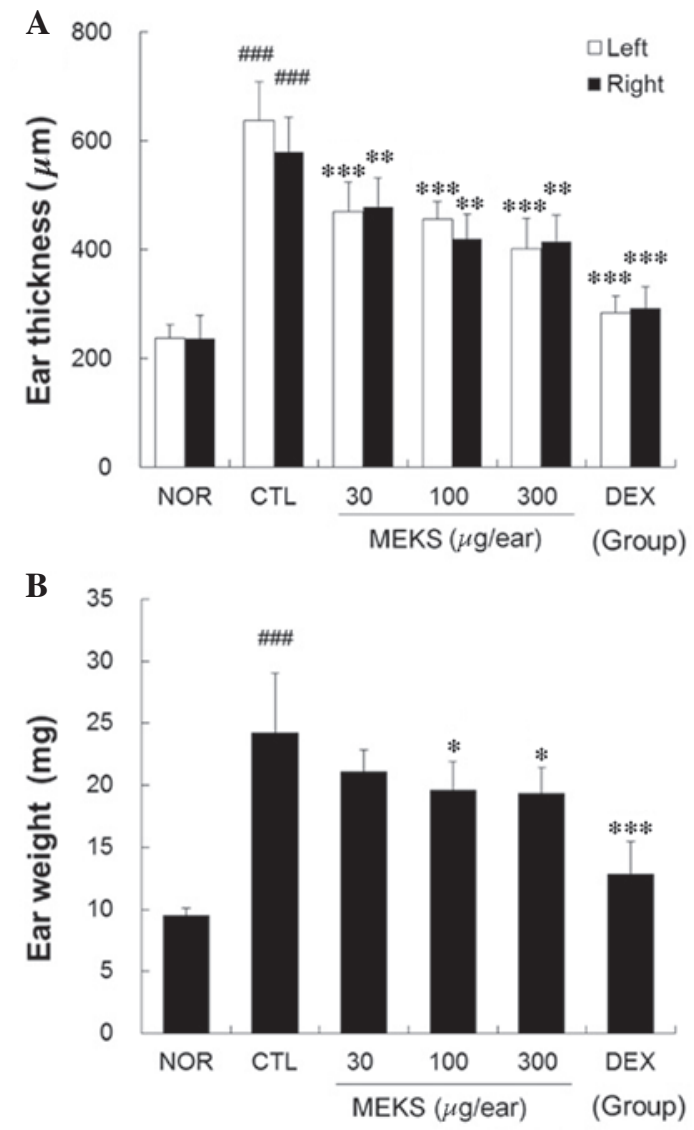

Figure 2. Effect of MEKS on ear thickness and weight in CD mice. Reduction of ear (A) thickness and (B) weight, in response to topical application of MEKS, was analyzed using vernier calipers and a microbalance on day 15. Values are presented as means \pm standard deviation. ${ }^{\# \# \#} \mathrm{P}<0.001$ vs. NOR ${ }^{*} \mathrm{P}<0.05,{ }^{* *} \mathrm{P}<0.01$ and ${ }^{* * * *} \mathrm{P}<0.001$ vs. CTL. CD, contact dermatitis; NOR, non-treated normal mice; CTL, non-treated CD mice; DEX, dexamethasone; MEKS, methanol extracts of Kochia scoparia dried fruit.

the NOR group demonstrated normal epidermal thickness and a smaller degree of immune cell infiltration into the dermis (Fig. 3A). Repeated application of DNFB induced small and large subcorneal and intraepidermal vesicles, diffused spongiotic changes and intercellular edema, which are characteristics of CD (Fig. 3B). Topical application of MEKS inhibited epidermal spongiosis in the inflamed tissue (Fig. 3C-E).

MEKS inhibited epidermal acanthosis and immune cell infiltration. Repeated application of DNFB induced acanthosis, hyperkeratosis and focal crust formation in the epidermis. In addition, DNFB induced diffuse acute and chronic immune cell infiltration, blood vessel dilation and perivascular eosinophil infiltration into the dermis. Topical application of MEKS effectively relieved epidermal acanthosis and immune cell infiltration in a dose-dependent manner (epithelial thickness: $\mathrm{P}<0.05,100 \mu \mathrm{g} /$ ear MEKS treatment; $\mathrm{P}<0.01,300 \mu \mathrm{g} / \mathrm{ear}$ MEKS treatment; $\mathrm{P}<0.001,75 \mu \mathrm{g} / \mathrm{ear} \mathrm{DEX}$ treatment. Immune cell infiltration: $\mathrm{P}<0.001,100$ or $300 \mu \mathrm{g} /$ ear MEKS treatment or $75 \mu \mathrm{g}$ /ear DEX treatment) as presented in Fig. 4.

$M E K S$ reduced expression levels of $T N F-\alpha, I F N-\gamma$ and $M C P-1$ in ear tissue samples of $C D$ mice. Marked increases in TNF- $\alpha$, IFN- $\gamma$ and MCP-1 production were observed in the CTL group. These increases were effectively reduced in a dose-dependent manner by topical application of MEKS (TNF- $\alpha$ and IFN- $\gamma: \mathrm{P}<0.05,100 \mu \mathrm{g} /$ ear MEKS treatment; $\mathrm{P}<0.01,300 \mu \mathrm{g} /$ ear MEKS treatment; $\mathrm{P}<0.001,75 \mu \mathrm{g} / \mathrm{ear}$ DEX treatment. MCP-1: $\mathrm{P}<0.01,300 \mu \mathrm{g} /$ ear MEKS treatment or $75 \mu \mathrm{g}$ /ear DEX treatment). Treatment with MEKS did not affect the production of IL-10, although DEX treatment reduced the IL-10 level significantly when compared with that of the NOR and CTL groups $(\mathrm{P}<0.05,75 \mu \mathrm{g} / \mathrm{ear} \mathrm{DEX}$ treatment) as presented in Fig. 5.

MEKS did not affect spleen/body weight ratio in CD mice. The effects of MEKS on enlargement of the spleen were estimated in terms of the spleen/body weight ratio. The spleen/body weight ratio in the CTL group was significantly elevated compared with the NOR group $(\mathrm{P}<0.001)$. Treatment with MEKS did not affect the spleen/body weight ratio in CD mice. However, this ratio was significantly reduced in the DEX group when compared with that of the CTL and NOR groups (Fig. 6; P<0.001).

\section{Discussion}

The present study demonstrated the anti-inflammatory effects of MEKS on CD. MEKS effectively prevented ear thickness and weight increases, as well as epidermal acanthosis, spongiosis and immune cell infiltration in inflamed tissues. In addition, the expression levels of TNF- $\alpha$, IFN- $\gamma$ and MCP-1 were dose-dependently reduced in response to MEKS. These findings indicate that MEKS treatment prevents the inflammatory reactions, which leads to the inhibition of ear thickness and weight increases in inflamed tissues.

Inflammatory reactions, such as immune cell infiltration and pro-inflammatory cytokine production, are important in the pathophysiology of $\mathrm{CD}$ and may serve as therapeutic targets. During progression of CD, IL- 1 and TNF- $\alpha$ markedly upregulate various chemokines, resulting in the recruitment of leukocytes (5). In addition, IFN- $\gamma$ is indicative of type 1 $\mathrm{T}$ helper skewing reaction of $\mathrm{T}$ cells and rapidly promotes the secretion of mediators, including chemokine ( $\mathrm{C}-\mathrm{X}-\mathrm{C}$ motif) receptor 3 agonist and $\mathrm{MCP}-1$, when it is present alone or together with TNF- $\alpha(13,14)$. The results of the present study are consistent with those of a previous study by Choi et al (12), who demonstrated that topical application of $K$. scoparia extract inhibited the expression of IL- $1 \beta$ and TNF- $\alpha$ messenger RNA. In the present study, the production of TNF- $\alpha$, IFN- $\gamma$, MCP-1 and IL-10 was evaluated using proteins obtained from samples of lysed tissues. Treatment with MEKS effectively decreased expression levels of TNF- $\alpha$ and IFN- $\gamma$ in inflamed tissues. MCP-1, also termed chemokine (C-C motif) ligand 2, is a small chemokine that belongs to the $\mathrm{CC}$ chemokine family, which recruits monocytes, $\mathrm{T}$ cells and dendritic cells to inflammatory sites $(15,16)$. In the present study, treatment with $300 \mu \mathrm{g} / \mathrm{ear}$ of MEKS significantly inhibited MCP-1 production in samples of ear tissue. These results indicate that the underlying mechanism of MEKS inhibition of immune cell infiltration involves decreasing MCP-1 production.

The effects of MEKS on CD were further demonstrated by histopathological analysis, which indicated that epidermal 
A

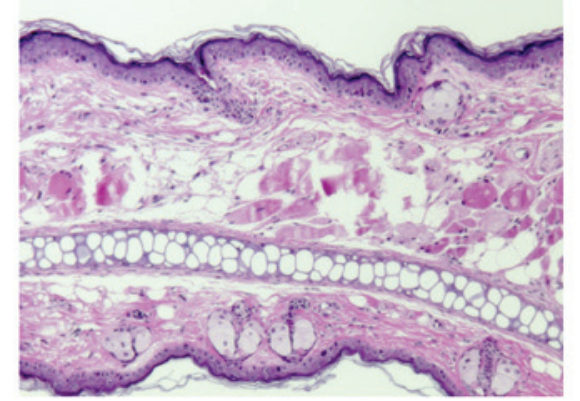

D

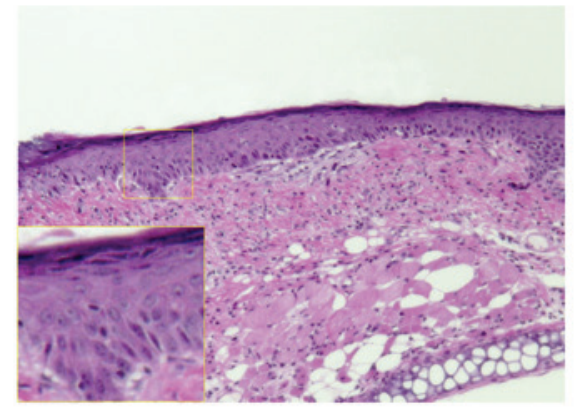

B

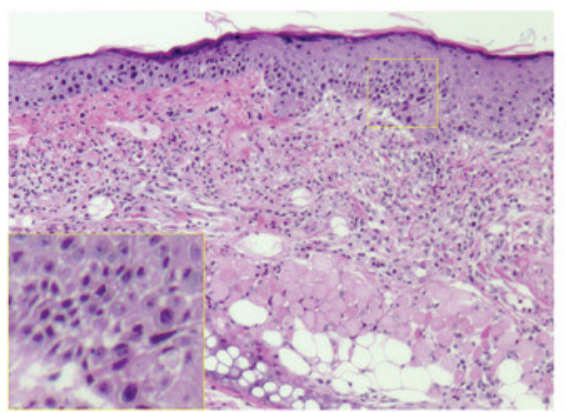

E

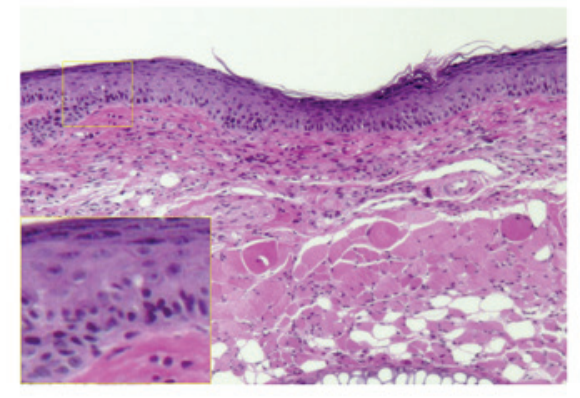

C

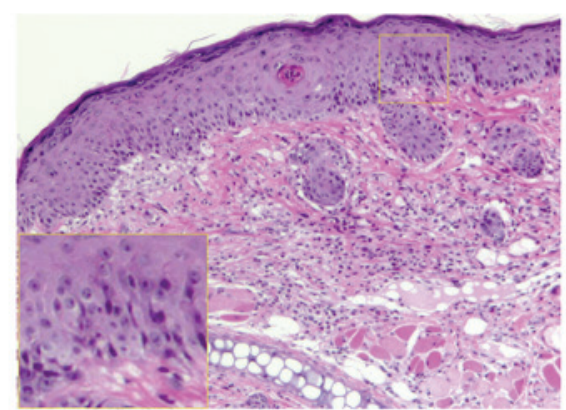

F

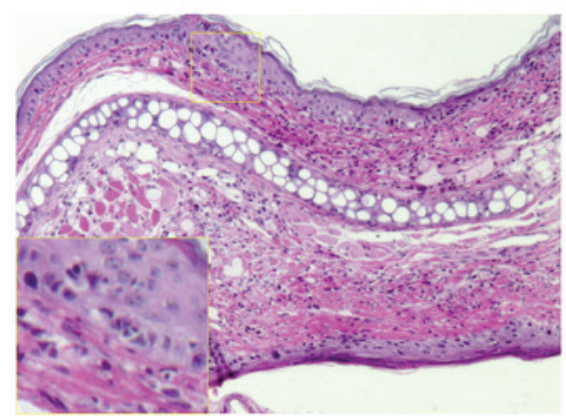

Figure 3. Effect of MEKS on histopathological changes in CD mice. Ear tissues were stained with hematoxylin and eosin and observed using a light microscope. (A) NOR group, (B) CTL group, (C) $30 \mu \mathrm{g} / \mathrm{ear}$ MEKS-treated group, (D) $100 \mu \mathrm{g} / \mathrm{ear}$ MEKS-treated group, (E) $300 \mu \mathrm{g} / \mathrm{ear}$ MEKS-treated group and (F) $75 \mu \mathrm{g} /$ ear dexamethasone-treated group. The insets represent spongiotic areas. Magnification, x200. MEKS, methanol extracts of Kochia scoparia dried fruit; CD, contact dermatitis; NOR, non-treated normal mice; CTL, non-treated CD mice.
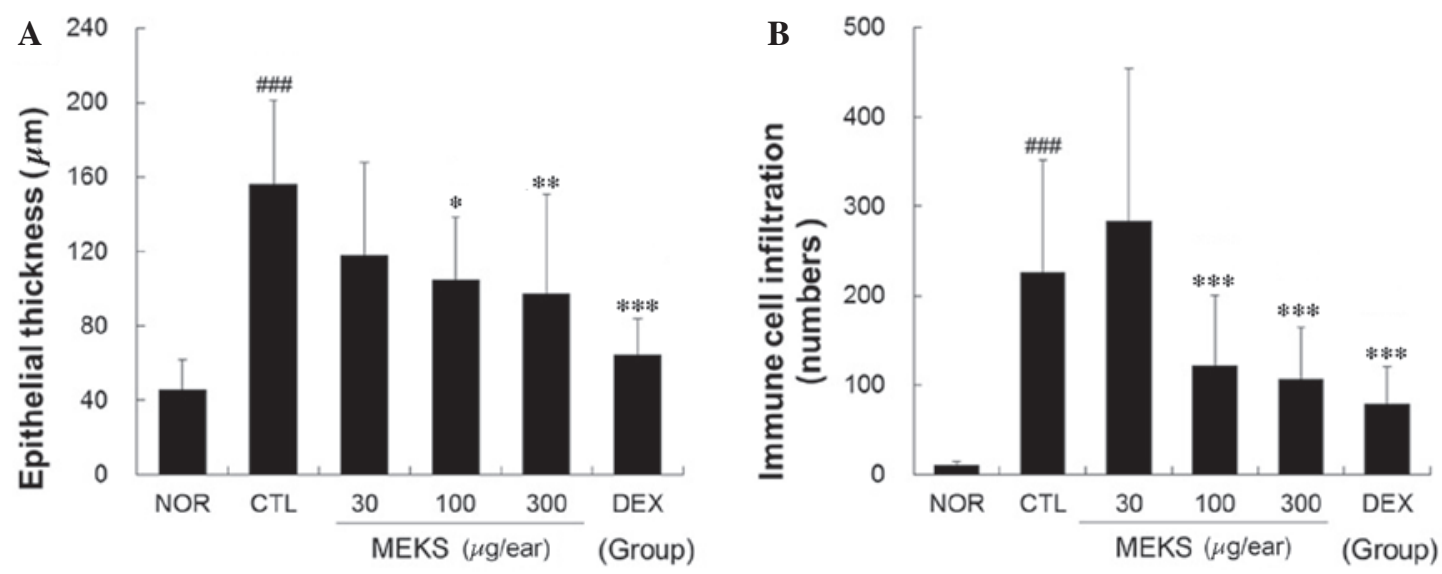

Figure 4. Effects of MEKS on epidermal acanthosis and immune cell infiltration in inflamed tissue. (A) The epithelial thickness and (B) number of infiltrated immune cells were observed under a light microscope. All values are presented as means \pm standard deviation. ${ }^{\# \# "} \mathrm{P}<0.001$ vs. NOR; ${ }^{*} \mathrm{P}<0.05$, ${ }^{* *} \mathrm{P}<0.01$ and ${ }^{* * * *} \mathrm{P}<0.001$ vs. CTL. MEKS, methanol extracts of Kochia scoparia dried fruit; DEX, dexamethasone; NOR, non-treated normal mice; CTL, non-treated contact dermatitis mice.

hyperplasia, spongiosis and immune cell infiltration were decreased by topical application of MEKS. Keratinocytes at the site of CD overexpress numerous cytokines and chemokines, such as TNF- $\alpha$, IFN- $\gamma$, MCP-1 and IL-10 (17-19). It has been previously reported that IFN- $\gamma$ is important in the development of skin hypertrophy, and TNF- $\alpha$ and MCP-1 function as stimulants of immune cell recruitment around blood vessels (17-19). In the present study, the topical application of MEKS inhibited the histopathological features of CD via suppression of TNF- $\alpha$, IFN- $\gamma$ and MCP-1 expression. These observations and the changes in cytokine production in the MEKS-treated tissue samples, suggest that MEKS is an anti-inflammatory agent against the Th1 skewing reaction, thus, reducing inflammatory reactions, such as epidermal acanthosis, spongiosis and immune cell infiltration.

DEX and MEKS prevented ear swelling, diminished epidermal acanthosis, spongiosis and immune cell infiltration and decreased the levels of TNF- $\alpha$, IFN- $\gamma$ and MCP-1 in ear tissue samples. MEKS did not affect the expression of IL-10, however, DEX significantly reduced the expression levels of all cytokines examined in the present study, including IL-10, which has a suppressive role in CD and atopic dermatitis (20). Treatment with DEX also led to a significant reduction in the spleen/body weight ratio when compared with the CTL 

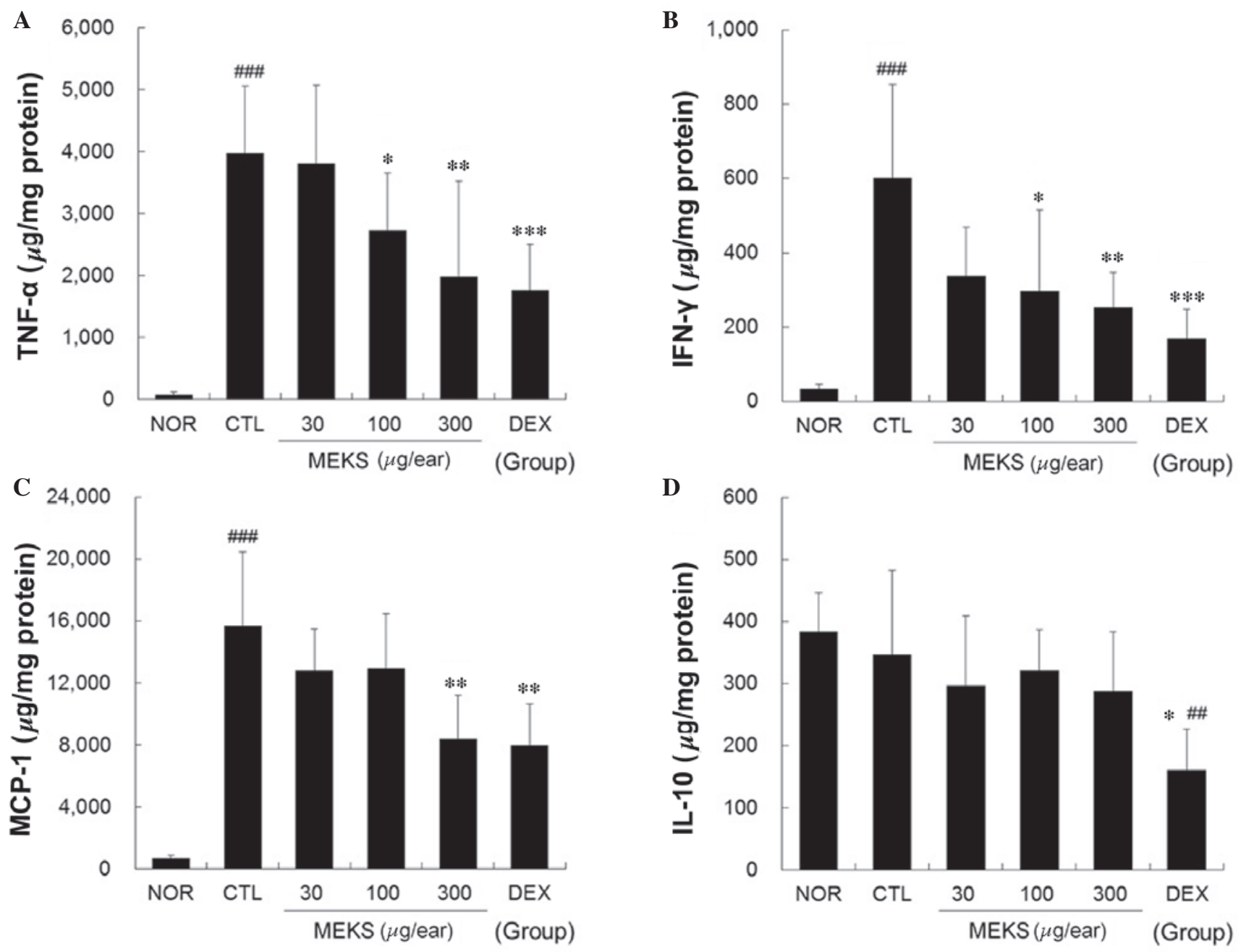

Figure 5. Effect of MEKS on expression levels of TNF- $\alpha$, IFN- $\gamma$, MCP-1 and IL-10 in CD mice. The expression levels of (A) TNF- $\alpha$, (B) IFN- $\gamma$, (C) MCP-1 and (D) IL-10 in the ear tissues were analyzed using the cytometric bead array method. A total of $50 \mu \mathrm{g}$ tissue lysates was used to measure the cytokine levels. All values are presented as means \pm standard deviation. ${ }^{\# \#} \mathrm{P}<0.01,{ }^{\# \# "} \mathrm{P}<0.001$ vs. NOR; ${ }^{*} \mathrm{P}<0.05,{ }^{* * *} \mathrm{P}<0.01$ and ${ }^{* * *} \mathrm{P}<0.001$ vs. CTL. TNF- $\alpha$, tumor necrosis factor- $\alpha$; IFN- $\gamma$; interferon- $\gamma$; MCP-1, monocyte chemotactic protein-1; IL-10, interleukin-10; NOR, non-treated normal mice; CD, contact dermatitis; CTL, non-treated CD mice; MEKS, methanol extracts from Kochia scoparia dried fruit; DEX, dexamethasone.

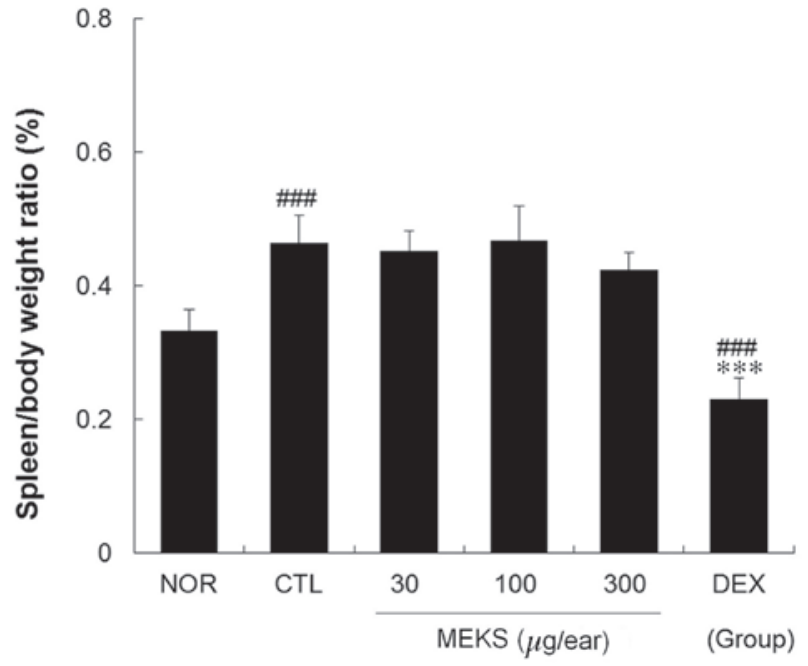

Figure 6. Effect of MEKS on spleen/body weight ratio in CD mice. Body and spleen weights were measured on day 15 and the spleen/body weight ratio was calculated. All values are presented as means \pm standard deviation. ${ }^{\# \# \#} \mathrm{P}<0.001$ vs. NOR; ${ }^{* * * *} \mathrm{P}<0.001$ vs. CTL. MEKS, methanol extracts of Kochia scoparia dried fruit; CD, contact dermatitis; NOR, non-treated normal mice; CTL, non-treated CD mice; DEX, dexamethasone. and the NOR groups, which is indicative of an immune reaction. These findings indicate that the underlying therapeutic mechanism of MEKS differs to that of DEX, particularly with regards to general immune suppression, which is one of the major side effects of corticosteroids, such as DEX.

The findings of the present study indicate that MEKS may be administered for the treatment of inflammatory skin diseases. In addition, the current study suggests that an anti-inflammatory mechanism of MEKS is involved in the inhibition of Th1 skewing reactions.

In conclusion, the present study demonstrated that MEKS reduces Th1 skewing reactions, such as production of TNF- $\alpha$, IFN- $\gamma$ and MCP-1, resulting in decreased epidermal acanthosis, spongiosis and immune cell infiltration. Repeated administration of MEKS resulted in anti-inflammatory reactions leading to the inhibition of ear swelling. The effects of MEKS were similar to those of DEX, however, no general immune suppression was observed in response to MEKS treatment. The findings of the present study indicate that MEKS may be used to reduce or replace corticosteroid use with relative safety. 


\section{Acknowledgements}

This research was supported by the National Research Foundation of Korea grant funded by the Korean government (MSIP; grant no. 2015R1A2A2A04005619).

\section{References}

1. English JS: Current concepts of irritant contact dermatitis. Occup Environ Med 61: 722-726, 674, 2004.

2. Usatine RP and Riojas M: Diagnosis and management of contact dermatitis. Am Family Physician 82: 249-255, 2010.

3. Streit M and Braathen LR: Contact dermatitis: Clinics and pathology. Acta Odontol Scand 59: 309-314, 2001.

4. Lee HY, Stieger M, Yawalkar N and Kakeda M: Cytokines and chemokines in irritant contact dermatitis. Mediators of Inflamm 2013: 916497, 2013.

5. Grabbe S and Schwarz T: Immunoregulatory mechanisms involved in elicitation of allergic contact hypersensitivity. Immunol Today 19: 37-44, 1998.

6. Kim NY, Lee MK, Park MJ, Kim SJ, Park HJ, Choi JW, Kim SH, Cho SY and Lee JS: Momordin Ic and oleanolic acid from Kochiae fructus reduce carbon tetrachloride-induced hepatotoxicity in rats. J Med Food 8: 177-183, 2005

7. Choi J, Lee KT, Jung H, Park HS and Park HJ: Anti-rheumatoid arthritis effect of the Kochia scoparia fruits and activity comparison of momordin lc, its prosapogenin and sapogenin. Arch Pharm Res 25: 336-342, 2002.

8. Lin YH, Chen YC, Hu S, Chen HY, Chen JL and Yang SH: Identifying core herbal treatments for urticaria using Taiwan's nationwide prescription database. J Ethnopharmacol 148 556-562, 2013

9. Shin KM, Kim YH, Park WS, Kang I, Ha J, Choi JW, Park HJ and Lee KT: Inhibition of methanol extract from the fruits of Kochia scoparia on lipopolysaccharide-induced nitric oxide, prostaglandin [correction of prostagladin] E2, and tumor necrosis factor-alpha production from murine macrophage RAW 264.7 cells. Biol Pharm Bull 27: 538-543, 2004.
10. Lee MY, Shin IS, Lim HS, Seo CS, Ha H and Shin HK: Kochia scoparia fruit attenuates allergic airway inflammation in ovalbumin (OVA)-induced murine asthma model. Inhal Toxicol 23: 938-946, 2011.

11. Matsuda H, Dai Y, Ido Y, Yoshikawa M and Kubo M: Studies on kochiae fructus. IV. Anti-allergic effects of $70 \%$ ethanol extract and its component, momordin Ic from dried fruits of Kochia scoparia L. Biol Pharm Bull 20: 1165-1170, 1997.

12. Choi YY, Kim MH, Lee JY, Hong J, Kim SH and Yang WM: Topical application of Kochia scoparia inhibits the development of contact dermatitis in mice. J Ethnopharmacol 154: 380-385, 2014.

13. Albanesi C, Cavani A and Girolomoni G: IL-17 is produced by nickel-specific T lymphocytes and regulates ICAM-1 expression and chemokine production in human keratinocytes: Synergistic or antagonist effects with IFN-gamma and TNF-alpha. J Immunol 162: 494-502, 1999.

14. Sebastiani S, Albanesi C, De PO, Puddu P, Cavani A and Girolomoni G: The role of chemokines in allergic contact dermatitis. Arch Dermatol Res 293: 552-559, 2002.

15. Carr MW, Roth SJ, Luther E, Rose SS and Springer TA: Monocyte chemoattractant protein 1 acts as a T-lymphocyte chemoattractant. Proc Natl Acad Sci USA 91: 3652-3656, 1994.

16. Xu LL, Warren MK, Rose WL, Gong W and Wang JM: Human recombinant monocyte chemotactic protein and other $\mathrm{C}-\mathrm{C}$ chemokines bind and induce directional migration of dendritic cells in vitro. J Leukoc Biol 60: 365-371, 1996.

17. Tung D, Cheung PH, Kaur P, Foreman O, Kavirayani A, Hain HS and Saha S: Anti-inflammatory and immunomodulatory effects of bortezomib in various in vivo models. Pharmacology 88: 100-113, 2011.

18. Fukuda S, Midoro K, Kamei T, Gyoten M, Kawano Y, Ashida Y and Nagaya H: Inhibition of allergic dermal inflammation by the novel imidazopyridazine derivative TAK-427 in a guinea pig experimental model of eczema. J Pharmacol Exp Ther 303: 1283-1290, 2002.

19. Gaffal E, Cron M, Glodde N, Bald T, Kuner R, Zimmer A, Lutz B and Tüting T: Cannabinoid 1 receptors in keratinocytes modulate proinflammatory chemokine secretion and attenuate contact allergic inflammation. J Immunol 190: 4929-4936, 2013.

20. Boyman O, Werfel T and Akdis CA: The suppressive role of IL-10 in contact and atopic dermatitis. J Allergy Clin Immunol 129: 160-161, 2012. 